\title{
Section introduction \\ Introduction to sessions on 'Endocrine therapy: where have we come from, where are we at and where are we going to?'
}

James $\mathrm{N}$ Ingle

Mayo Clinic, First Street SW, Rochester, MN 55905, USA

Corresponding author: James N Ingle, ingle.james@mayo.edu

Published: 18 December 2008

This article is online at http://breast-cancer-research.com/content/10/S4/S15 (c) 2008 BioMed Central Ltd

Two of the clinical sessions focused on endocrine therapy of breast cancer. It is clear that whereas endocrine therapy represents a crucial modality in breast cancer management, with substantial success to date, there is still much to be learned and great potential for increasing its value to patients. This therapeutic gain will probably come from a better understanding of resistance to endocrine therapy, both de novo and acquired; better selection of the right endocrine agent for the right patient; and better management of adverse events and noncompliance. Importantly, endocrine therapy is an important backbone upon which targeted therapy combinations will be built. These sessions addressed many of these important issues.

Professor Craig Jordan [1] considered the most important lessons learned from studying the action of oestrogen on breast cancer cells as a basis for future research. He reviewed the mechanisms of action of oestrogen through the oestrogen receptor (ER) and the complexity of these interactions. A new concept of evolution of resistance to selective ER modulators was considered, and it was noted that oestradiol can kill both selective ER modulator stimulated and aromatase inhibitor (AI) resistant cells. The current status of endocrine therapy in the adjuvant and prevention settings was reviewed, and angiogenesis inhibition and apoptosis induction were considered as future promising areas of research.

Professor James Ingle [2] emphasized the fact that variability exists in how patients respond to endocrine therapy, and that there is variability in terms of the effects of endocrine agents on end organs and adverse events. He reviewed the concepts of pharmacogenetics, pharmacogenomics, pharmacodynamics and pharmacokinetics. The field of pharmacogenetics/genomics addresses the genetic factors of the host and has the goal of identifying the right drug at the right dose for the right patient. It is clear that the field is at an early stage for endocrine therapy of breast cancer, but there is emerging evidence that cytochrome P450-2D6 metabolizer status is of
Breast Cancer Research 2008, 10(Suppl 4):S15 (doi:10.1186/bcr2175)

predictive value in patients considering tamoxifen therapy. Further validation of these findings is needed before there can be widespread acceptance. The field is at an earlier stage of development for the Als but research is ongoing.

Professor Daniel Hayes [3] addressed the issue of markers of endocrine sensitivity. He considered the topics of endocrine resistance and definitions of tumour markers, including levels of evidence and the importance of determining whether a marker is prognostic or predictive. The ER was considered to be the most important predictive factor for endocrine therapy, and he reviewed the weaknesses that exist with this marker. Candidate markers most likely to complement the ER were considered.

Professor William Miller [4] addressed the identification and mechanisms of endocrine resistance that are central to advancing the efficacy of endocrine therapy in breast cancer. $\mathrm{He}$ considered endocrine resistance from the standpoints of primary and acquired resistance, and categories of clinical, proliferative and molecular resistance. The mechanisms of resistance were considered in terms of inherent hormone insensitivity, nonendocrine growth signalling, ER activation/ hypersensitivity and inefficient/compromised therapy. Importantly, he reviewed our current understanding of means to identify these different categories of resistance.

Professor Stephen Johnston [5] addressed the status of research into targeting the ER and signalling pathways simultaneously. The clinical progress to date was summarized, considering both studies that revealed negative results as well as those that revealed positive results. In particular, trials with Als and epidermal growth factor receptor/HER-2 (human epidermal growth factor-2) targeted therapy appear to offer substantial potential for progress. He presented the concepts of 'vertical growth factor pathway blockade' and 'horizontal pathway blockade' as being important approaches to targeting multiple signalling pathways. 


\section{Competing interests}

The author declares that they have no competing interests.

\section{Acknowledgement}

This article has been published as part of Breast Cancer Research Volume 10 Supplement 4, 2008: Controversies in Breast Cancer 2008. The full contents of the supplement are available online at http://breast-cancer-research.com/supplements/10/S4

\section{References}

1. Jordan VC: By looking back we can see the way forward: enhancing the gains achieved with antihormone therapy. Breast Cancer Res 2008, 10(Suppl 4):S16.

2. Ingle JN: Pharmacogenetics and pharmacogenomics of endocrine agents for breast cancer. Breast Cancer Res 2008, 10(Suppl 4):S17.

3. Hayes DF: Markers of endocrine sensitivity. Breast Cancer Res 2008, 10(Suppl 4):S18.

4. Miller WR: Identification and mechanisms of endocrine resistance. Breast Cancer Res 2008, 10(Suppl 4):S19.

5. Johnston SRD: Integration of endocrine therapy with targeted agents. Breast Cancer Res 2008, 10(Suppl 4):S20. 